\title{
Converting System Limits to Market Signals
}

\author{
Fernando L. Alvarado, Fellow, IEEE
}

Invited Paper

\begin{abstract}
This paper compares methods for converting system limits into market signals. One classification of methods is according to reliability driven (TLR and similar) versus market driven (LMP and similar) methods. A second classification is according to direct versus indirect methods. Direct methods deal with individual limits and constraints. Indirect methods include various ways of converting one type of limit to another, equivalent limit for purposes of making the handling of the limit more expeditious. An example of an indirect method is the conversion of a voltage limit to either a flow limit or an interface limit. Another example is the use of flow limits on interfaces as surrogates for stability limits. These transformed limits are often represented by nomograms. Conversion of one type of limit to another and the construction of nomograms has the advantage of reducing the problem of imposing system limits within a market context to a "previously solved" market problem. If a market already has learned how to cope with an import limit into a load pocket, conversion of a voltage limit into a load pocket import limit makes it easy for a market to react and respond to the condition. However, any conversion from one type of limit to another entails an approximation. This paper discusses the nature of some of these approximations.
\end{abstract}

Index Terms-ATC, deregulation, flowgates, interface limits, LMP, locational marginal pricing, nomograms, stability limits, transfer limits, transmission limits, voltage limits.

\section{NOMENCLATURE}

ATC

Available transfer capability is a measure of the transfer capability remaining in the physical transmission network for further commercial activity over and above already committed uses. Refer to [13] for details.

Congestion Condition that results in reaching or violating a system capability limit. At a minimum, a system operating under congestion conditions cannot permit congestion conditions to worsen.

Contingency Outage of a component such as a line, generator, or transformer. Often system limits are enforced on all credible contingencies.

FGR Flowgate rights, the right to receive compensation based on the congestion of a particular limiting facility.

Manuscript received January 10, 2003. This work was supported in part by the Consortium for Electric Reliability Technology Solution (CERTS) under Department Of Energy Interagency Agreement DE-AI-99EE35075 with NSF and in part by PSerc, the Power Systems Engineering Research Center.

The author is with the University of Wisconsin and Christensen Associates, Madison, WI 53705 USA (e-mail: alvarado@engr.wisc.edu).

Digital Object Identifier 10.1109/TPWRS.2003.810669
Flow limit

Flowgate

FTR

Interface

LMP

Nomogram

\section{OASIS}

OPF

PTDF

Reserves

Stability limit
Maximum amount of power that can be transmitted by a line, cable, or transformer. See [5].

Any line or transformer, or set of lines and transformers where there is a restriction on the total power that may flow through the set. Firm (or financial) transmission rights, the right to receive compensation for the price difference resulting from congestion between two points no matter what the limiting facility or facilities might be.

A set of lines usually connecting two areas of the system or defining a load or generation "pocket." Often limits (particularly stability limits) are associated with interface flows rather than with individual line flows.

Locational (or nodal) marginal price, the cost of delivering one additional MW of electricity to a given location while respecting all system limits in effect.

A nomogram is a two-dimensional representation of the operational capabilities of a system when there is a tradeoff between two decision variables, More generally, a nomogram is any representation of the interaction between two or more decision variables.

Open access same time information system, a way of posting ATC information for all to see.

Optimum power flow, a problem formulation intended to determine the optimal dispatch for a system given a specific system configuration, subject to given limits and constraints. The Lagrange multipliers associated with the OPF solution can be interpreted as nodal prices (see LMP).

Power Transfer Distribution Factor, the impact on a given flowgate of changing the injection at a location by $1 \mathrm{MW}$ (relative to a reference location).

Amount of active (and sometimes reactive) power needed so that the system can continue operation after a contingency. The reserve level is usually selected on an area by area basis according to agreed-upon criteria. Inability to operate properly as a result of either oscillatory conditions or the threat of loss of synchronism if a contingency occurs. 


\section{TLR}

Voltage limit
Transmission loading relief, a method for curtailing transactions based on rules rather than market signals.

Inability to operate properly as a result of either low voltage or proximity to voltage collapse conditions. Voltage limits are usually the result of insufficient reactive power.

\section{INTRODUCTION}

$\mathbf{T}$ HE INTENT of this paper is to provide a unifying perspective on the topic of how limits affect markets. The paper discusses various methods and technologies for converting system limits into market signals. The various system limits considered include flow limits, interface limits, active and reactive power reserve requirements, voltage requirements, stability limits, energy balance requirements (including the need to control system frequency), and a multitude of other "practical" system limits. The paper describes both direct and indirect means for creating "market signals" out of all these limits. The direct methods use the limits themselves in the creation of the signals, whereas the indirect methods include various ways of converting one type of limit into an (approximately) equivalent limit for the purpose of making the handling of the limit more expeditious.

Once limits are established, the manner in which they are revealed to the market can vary. One way of issuing signals to a market is by disclosing and imposing the limits on all transactions and operational conditions based on rules having to do with priority, order of transactions, size of transaction, the use of a reservation system or other such nonpricing technique. We call this the reliability-driven approach. The second alternative to limit enforcement is by the use of price signals that ensure that the limit is not violated by ascribing economic value to the limit. We call this the market-driven alternative.

Within market-driven methods for enforcing limits, there are at least two (not necessarily incompatible) ways of dealing with limit enforcement. One is the conversion of the limit into nodal price signals of direct interest to market participants. The second alternative is to price the limit itself and to calculate the contribution to this limit of any transaction or action among market participants.

The direct methods are closely linked to the notion of optimum power flow. In particular, the Lagrange multipliers associated with the nodal power balance equations at the solution point are, in fact, the nodal prices. Within direct methods some can be considered exact and some include deliberate approximation and/or aggregation. The indirect methods include various ways of converting one type of limit to an equivalent limit. An example of such limit is the conversion of a voltage limit to a "load pocket import limit." These converted or surrogate limits are often represented or described by "nomograms." Conversion of one type of limit to another and the construction of nomograms has the advantage of reducing the problem of imposing system limits to a previously solved market problem. If a market already has learned how to cope with an import limit into a load pocket, conversion of a voltage limit into a load pocket import limit makes it easy for a market to react and respond to the con- dition. However, conversion from one type of limit to another can result in incorrect market signals. This is illustrated in the Appendix, and also in [1].

\section{RELIABILITY DRIVEN VERSUS. MARKET DRIVEN LiMITS}

The first classification on how to incorporate limits is along the lines of whether a reliability criterion is established to monitor and enforce limits (irrespective and independently of a market), or whether the market is designed and organized to explicitly consider system limits in its design. ${ }^{1}$

\section{A. Reliability Driven Limit Enforcement}

In the reliability-driven model of market operation, trade is enabled by posting available capacities and requirements of the system for those engaged in commercial activities and then setting up a reservation system to allocate and approve trades. The system operator retains the authority to do administrative overrides of trades (including previously approved trades) when they impair system reliability.

The scenario for limit enforcement based on the reliabilitydriven viewpoint is as follows:

- for every important transaction, an ATC value is calculated. ATCs can change due to interactions among transactions. Once a transaction is enacted, the ATCs for other transactions may be affected. The exact definition of ATC is system-dependent (the NERC web site www.nerc.org lists over two dozen slightly different implementations of ATC for various systems);

- transfer capabilities are posted on an OASIS;

- tariff-based reservations of available transfer capacity for specific transactions are made and approved;

- ATCs are updated as needed;

- transactions that violate security rules are not authorized;

- if limit violations occur during operations, a method for curtailment of transactions (TLR) goes into effect;

- an extension to TLR is Market Redispatch (MRD). MRD refers to the scheduling of an alternative transaction to be used by the dispatcher in lieu of a curtailment of a problematic transaction when and if this alternative transaction would attain the goal of congestion relief.

The NERC transmission loading relief (TLR) procedure is an Eastern interconnection-wide procedure to allow the reliability coordinators to 1) respect transmission service reservation priorities, and 2) mitigate potential or actual operating security limit violations. The procedure is based on a formula developed by NERC. The procedure is documented in detail along with examples in [12].

The TLR procedure is in theory capable of addressing most congestion situations. In practice, there are a number of problems with TLR. These problems are discussed in [3]:

- the TLR system can be "gamed" by, for example, by overscheduling of transactions;

- curtailment process does not take into consideration desirable counterflows;

${ }^{1}$ For an expanded discussion of the issues in this section, refer to [7]. 
- even when MRD is used, it is hard to optimize system operation;

- TLRs have limited ability to control transmission problems in view of the limited data used in TLR analysis.

While the TLR procedures are common practice and are deployed in the U.S. Eastern interconnection, an examination of the TLR Logs for actual curtailments suggests that the use of such procedures is uncommon in systems where locational marginal prices are used as the primary means for congestion management.

\section{B. Market-Driven Limit Enforcement}

In the market-driven view of system operations, the operator makes relatively aggressive use of market signals and prices and uses markets as much as possible to assure reliability. The idea is simple: any limit imposes a constraint on the market. When a limit is not binding, it is simply ignored. Whenever a limit becomes binding, it is incorporated into the market as an active constraint. The result is invariably that the prices seen by the market become differentiated (usually by location, but also by time period). By definition, the enforcement of limits is done optimally in a manner that best attains lowest cost while enforcing every limit.

\section{PhysicAl Rights Versus LMP Versus FGR}

In cases where the diversity in nodal prices can be traced to a well-defined small set of predictable limits, it is possible to establish a set of property rights for the use of individual congested facilities. The price for use of these facilities is their shadow price (or their Lagrange multiplier in an OPF solution). This price corresponds to the benefit of expanding the capacity of the facility by $1 \mathrm{MW}$. A system of property rights based on rights on individual congested facilities works best in radial situations where the number of facilities that can congest is relatively small and predictable. In more general situations, there are three ways of awarding property rights: Physical Rights, LMPs, and FGRs.

Property rights to limiting facilities can be implemented using a physical rights approach where the flows that a scheduled transaction produces through a constrained facility are determined according to the PTDFs, and the transaction must be backed by an appropriate portfolio of rights thief the transaction involves the use of a congested facility.

In theory, it can be shown that the value of the FTRs should converge to the value of the portfolio of physical rights that are necessary to support a specific transaction under congestion conditions. A physical rights approach may require the acquisition of rights on many potentially congested paths, so a simplification is made requiring that only a small predefined "commercially significant" subset of flowgates be addressed. Moreover, the exercise of physical rights requires much lastminute maneuvering to assure that rights are used and not lost, which adds to the complexities of system operation. In addition, many of the actions required by the physical rights approach may be in conflict with actions that the operator may wish to take in order to ensure real-time security; in other words, the physical rights approach to markets may be incompatible with the operator's need to control physical assets for security reasons.
An alternative way of awarding rights to congested facilities is by means of financial congestion rights, by establishing rights based on LMPs. It is not our intention to describe the fundamentals of LMPs or why they are often the preferred way to grant rights. For details on the approach, refer to [8], [9], or [11]. Suffice it to say that these rights correspond to the right to collect money anytime a price differential between the two nodes between which the rights have been purchased becomes nonzero. This is the approach in use at $\mathrm{PJM}^{2}$ and New York ${ }^{3}$ nowadays. If rights are not oversubscribed, the amount of congestion rent collected by the system operator should never be lower than the amount necessary to pay out for these congestion rents, thus the system operator is indifferent as to the holder of the rights. One advantage of this approach is that it permits spatial hedging for any party interested in hedging a transaction that may be affected by congestion or a system limit of any sort. The price paid for this right should, in principle, reveal the value of all the transmission network congested facilities. The system is simple to administer but requires "centralized" determination of prices.

Another approach to pricing transmission is based on flowgate rights (FGRs), in which parties acquire financial rights to specific flowgates. This approach represents a midway point between the physical rights and the FTR approaches. As in the case of physical rights, the acquisition of FGRs is based on distribution factors for flowgates that have been determined to be commercially significant. However, settlements are based on the actual marginal value of capacity on the flowgate at the time of congestion (i.e., the shadow prices on the constrained facilities). Under this setup, last-minute scheduling and operation are left to the operator, and all scheduled transactions are charged a transmission fee for the flows they induce on the congested facilities; this fee is equal to the corresponding shadow price of these facilities. A transaction that is backed by the proper portfolio of FGRs will collect settlement revenues that will exactly offset its transmission fees. In practice, however, a transaction would be covered by only a limited set of flowgate rights that only approximately track changing distribution factors, which would leave some residual congestion risk exposure unhedged. Such flexibility decouples operational decisions from the settlement issues associated with transmission rights.

There is a "fundamental mathematical equivalence" between FTRs, FGRs, and physical rights. In other words, under ideal circumstances all three should lead to the exact same set of prices and incentives, although based on the foregoing discussion it should be clear that the "mechanics" of all three approaches can differ substantially and lead, in practice, to quite different prices and incentives.

For a more detailed explanation of all three types of rights (with emphasis on contrasting FGRs and FTRs), refer to [15] and [16]. For additional examples, formulas, and details of the approach, see also [17].

All three types of rights can be handled as either obligations (in which case a party owning the rights may owe money if the prices "reverse") or as options (where no obligation to pay is necessary). Necessarily, the cost of an obligation will be either

\footnotetext{
2For extensive information on LMP's in PJM refer to web site www.pjm.com, particularly under the section entitled "PJM Transing."

${ }^{3}$ For details on the New York market, refer to NYISO's "Market Participant's User's Guide," June 2001 revision, under the topic of "LBMP."
} 
equal or lower than the cost of an option. Options are best implemented as derivative products, not as fundamental market components, but Texas and California have elected to use options ${ }^{4}$ for the handling of transmission rights. For a detailed description of unique aspects of the ERCOT and California, refer to [14].

\section{VOLTAGE LIMITS}

Voltages and voltage regulation are key to the functioning of the system. We describe three ways of addressing voltage problems within a market environment

\section{A. Uplift Payments}

Whenever a resource (such as a generator) is forced online as a result of the need for reactive power support, the generator is given a side payment that makes it "whole." A generator in this category should be given the opportunity to derive a sufficient profit to perform this function. By design, a generator payment that is made as an uplift does not "set the price" for power (active or reactive). Instead, the cost of making this side payment becomes part of an "uplift" that must be allocated.

\section{B. Voltage Limits Replaced With Flow Limits}

We call this the "nomogram" approach, because it results in the "mapping" of one type of limit onto another. Once the replacement is done and an equivalent (active power) flow is established, then the problem is reduced to a "conventional" congestion problem. Under these conditions, pricing of the resulting congestion can be done using ordinary congestion pricing methodologies. The result will generally be that a generator comes online in the desired region as a result of the higher prices, and the desired voltage regulation control is attained. If the generator also sets the marginal price for the node where it is activated (as it should be the case in a nodal or even in a zonal pricing system), the effect will be quite different than in the case of side-payments.

When replacing a voltage limit with a flow limit, there may be many choices as to which if the "right" flow limit to use. As the Appendix illustrates, the precise choice of which limit is used as a surrogate has implications, not on the solution point itself, but on the nodal prices that are posted for various nodes.

\section{Voltage Limits Treated Directly as Constraints}

The result of this type of limit is that the Lagrange multiplier associated with the voltage itself now has a value. The voltage itself is not, however, directly controllable. Even in voltage-controlling generators, the voltage is controlled by adjusting reactive power injections (and to a lesser extent the active power injections) at any of a number of nodes. Thus, a nodal price can be associated with any nodal active power injections as a direct consequence of a voltage limit. Likewise, a nodal price can be associated with every reactive power injection at every node as a result, if one wishes to do so. The manner in which this occurs is as Lagrange multipliers in the solution of an (OPF).

\footnotetext{
${ }^{4}$ This means that FTRs only have value if the congestion is in the direction that was purchased, and there is no "obligation" or risk associated with a price difference in the "opposite" direction.
}

Results in [1] indicate that the manner in which voltage limits are handled can have an effect on markets. In particular, nodal prices depend on what constraints and limits are assumed for the operating point. Identical operating points can give different nodal prices. The common practice of using nomograms where a flow limits is used in lieu of a voltage limit can result in prices that depart from the correct ones.

On the other hand, starting a generator in order to meet reactive and voltage constraints results in an "uplift" cost and lowers the marginal energy cost, also distorts prices.

\section{Simultaneous Flow And Stability Limits}

The desire to simplify market signals often results in a reduction in the number of presumed limiting constraints. Such efforts often lead to the replacement of a set of individual flow limits with a single limit on a corridor or interface. They also lead to the replacement of most stability limits with corresponding flow limits.

A corridor or interface limit can be used as a surrogate for the enforcement of more than one individual line limit in situations where these line limits are highly correlated and where the important information is not which limit has been reached but the fact that one of the limits has been reached. They are also quite commonly used to express stability limits between regions. In stability-limited cases, the use of a corridor limit is perhaps more natural than a limit on any individual line. Of particular interest is to contrast, for any specific system, the extent to which individual line flow limits can be "subsumed" by a single corridor or interface limits. In cases where the corridor limit has good correlation with the flow on several individual lines, it becomes possible to substitute many individual line limits with a single corridor limit, thereby simplifying the problem. However, in cases where there is poor correlation between the two, it is better to use individual line limits. This decision must be system-specific.

Safe transfer regions, whether for purposes of stability limit representation or for other purposes, can be captured by a nomogram that describes the interaction between two or more limits in a graphic manner. Nomograms for stability limits generally correspond to a specific base case, topology, and location of the source/sink buses. As conditions change, so does the nomogram. In particular, the location of source and sink can have a significant impact on the nomogram. Also, the use of flow control devices fundamentally alters the PTDFs for the system, and consequently affects the determination of flow limits. For additional information on these topics, refer to [2].

The use of flow or corridor limits to express stability limits, although in widespread use and fully justifiable for practical reasons, may itself be subject to errors in the sense that it represents a surrogate limit. The extent to which a flow limit or set of flow limits may or may not be a good surrogate from which to derive price signals for a system is not known.

\section{CONCLUding REMARKS}

Every physical limit has a nonzero marginal value to the system, otherwise it would not be limiting. Thus, markets must concern themselves with the impact of system limits. Since 
most electricity markets like to deal almost exclusively with the "main" quantity of interest (i.e., energy), it is often desirable to translate all limits into energy or flow limits. Furthermore, the desire for market transparency and simplicity often is translated into the desire to have as few limits and as little complexity as possible. Thus, simplified representations of system limits (captured in the form of nomograms) are often used. In addition, the desire to have reliability play a predominant role often results in a viewpoint that can be describes as a "reliability-first" philosophy. This paper has described and contrasted various ways of approximating limits of various types.

It is the opinion of this author that the use of LMPs and FGRs are simpler and less open to gaming than the use of physical rights reservation systems-based systems for awarding rights to congested physical facilities. This opinion has been formed after qualitative observation of the PJM, NYISO, and California markets and the inherent simplicity of LMP rules.

The use of surrogate limits (such as the use of flow limits in lieu of voltage limits) should be done with great care, since it has the tendency to distort system prices. Overall, this author recommends that, whenever possible, system limits should not be approximated and that the way in which limit information should be conveyed to the market should be as locational prices for both active and reactive power at every node in the system. Reserves, day ahead prices, reactive power and energy balancing services should be nothing more than derivative products on the real-time market for electricity fully incorporating limits and locational effects.

\section{APPENDIX}

This appendix illustrates the notion that when one replaces one limit with another, the solution point may in fact be identical, but the marginal prices can be different, depending on what one assumes the limit is. A different price at a node signifies a different economic incentive, and can lead to different actions taken based on such information.

To illustrate how identical solutions can give rise to different nodal prices, consider the four-bus system illustrated in Fig. 1. Assume that there is a voltage problem at bus 4 (where the load is) that gives rise to the need to take action. The action taken to prevent this problem can be any of the following cases:

1) declare bus 4 to be a load pocket and limit the imports into bus 4 by limiting the total flow across the $2-4$ and 3-4 interface;

2) limit the imports into bus 4 by controlling the flows across the 1-2 and 1-3 interface;

3) limit the imports into bus 4 by limiting any one line, such as limiting the flow on line 1 (from 1 to 2 );

4) directly impose a limit on the voltage at bus 4 along with a limit on the reactive power that can be supplied by bus 4 .

Each of these scenarios gives rise to different marginal prices. These prices are shown in Table I. Observe that the prices for nodes 2 and 3 can differ substantially.

In other words, the exact same operating point can give rise to different price patterns. It is thus important to agree on precisely what the limits are. Furthermore, although not illustrated here, if the pricing of reactive power is made explicit, the results can change further.

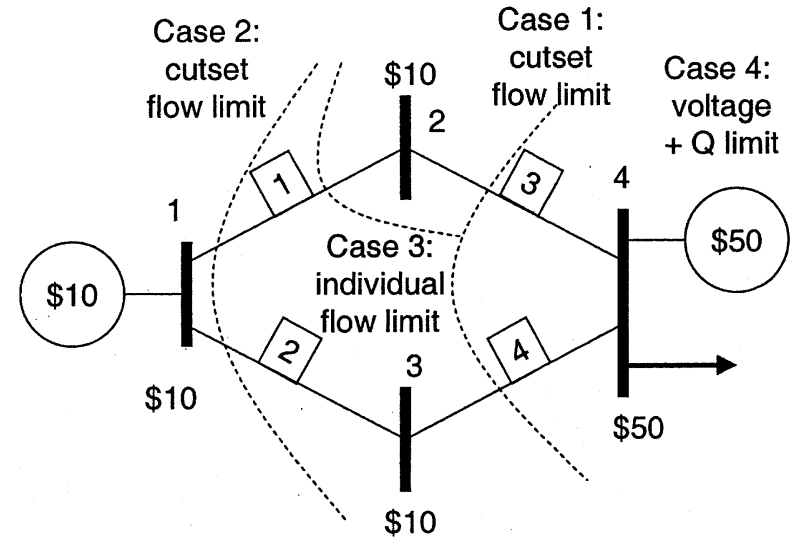

Fig. 1. Four node system. Lines modeled as 0.1-p.u. reactances. Voltages at buses 1 and 4 are 1 p.u. Bids are as shown. Assume that bus 4 has voltage "problems." Four alternatives for handling the voltage problem (illustrated) lead to the same solution but different prices.

TABLE I

NODAl PRICES FOR EACH OF THE FOUR CASES

\begin{tabular}{l|c|c|c|c}
\hline & Node 1 & Node 2 & Node 3 & Node 4 \\
\hline Case 1 & 10 & 10 & 10 & 50 \\
\hline Case 2 & 10 & 50 & 50 & 50 \\
\hline Case 3 & 10 & 70 & 30 & 50 \\
\hline Case 4 & 10 & 20 & 20 & 50 \\
\hline
\end{tabular}

\section{ACKNOWLEDGMENT}

The author gratefully acknowledges the contributions of $\mathrm{P}$. Sauer, F. Mancilla-David, and J. Zuluaga for their work on the project and Shmuel Oren and Rajesh Rajaraman for discussions and ideas that led to the improvement of the notions in this paper.

\section{REFERENCES}

[1] J. Zuluaga and F. L. Alvarado, "Voltage constraints and power nomograms," in Proc. 2002 Trans. North Amer. Power Symp., Phoenix, AZ, Oct. 2002.

[2] F. Mancilla-David and F. L. Alvarado, "Nomograms for simultaneous transfer capability," in Proc. 2002 Trans. North Amer. Power Symp., Phoenix, AZ, Oct. 2002.

[3] R. Rajaraman and F. L. Alvarado, "Inefficiencies of NERC's transmission loading relief procedure," in The Electricity J. New York: Elsevier, Oct. 1998, pp. 47-54.

[4] CIGRÉ Study Committee 38 Task Force 38-05-07, Methods and Tools for Costing Ancillary Services: F. L. Alvarado, Convenor, Dec. 2000.

[5] H. P. St. Clair, "Practical concepts in capability and performance of transmission lines," presented at the AIEE Pacific General Meeting AIEE Trans. Power Apparat. Syst., Vancouver, BC, Canada, Sept. 1-4, 1953, Paper 53-338.

[6] S. Wunderlich and R. O'Connell, "An inter-area transmission and voltage limitation (TVLIM) program," IEEE Trans. Power Syst., vol. 10, no. 3, Aug. 1995.

[7] F. L. Alvarado and S. Oren (2002, May), "Transmission system operation and interconnection," in National Transmission Grid Study Issue Papers: U.S. Department of Energy [Online]. Available: http://www.energy.gov/NTGS/ISSUE_1.PDF.

[8] R. E. Bohn, M. C. Caramanis, and F. C. Schweppe, "Optimal pricing in electrical networks over space and time," Rand J. Econ., vol. 15, pp. 360-376, 1984.

[9] W. W. Hogan, "Contract networks for electric power transmission," $J$. Regulatory Econ., vol. 4, pp. 211-242, 1992. 
[10] S. Greene, I. Dobson, and F. L. Alvarado, "Contingency ranking for voltage collapse via sensitivities from a single nose curve," IEEE Trans. Power Syst., vol. 14, pp. 232-238, Feb. 1999.

[11] R. Rajaraman and F. L. Alvarado, "Managing transmission risk: The theory of spatial hedging and arbitrage,", PSerc Rep. 98-27, Univ. Wisconsin.

[12] NERC Operating Manual Appendix 9C1. (2002, Oct.) Transmission Loading Relief Procedure-Eastern Interconnection. [Online]. Available: www.nerc.org

[13] NERC. (1996, June) Available Transfer Capability: Definitions and Determination. [Online]. Available: www.nerc.org

[14] W. W. Hogan, "Coordination for competition: Electricity market design principles," in Public Utility Commission of Texas Workshop on ERCOT Protocols, Austin, TX, Feb. 15, 2001.

[15] L. Ruff, "Flowgates, contingency-constrained dispatch, and transmission rights," The Elect. J., Jan./Feb. 2001.

[16] H.-p. Chao, S. C. Peck, S. Oren, and R. Wilson, "Flow-based transmission rights and congestion management," Elect. J., Oct. 2000.

[17] H.-p. Chao, "Flow-based rights for congestion management," presented at the MEET 2000 Workshop, Stanford, CA, Aug. 17-19, 2000.
Fernando L. Alvarado (F'98) was born in Lima, Peru, on September 15, 1945 He received the P.E. and B.E.E. degrees from the National University of Engineering, Lima, Peru, and received the Masters degree from Clarkson University, Potsdam, NY, in 1969, and the Ph.D. degree in electrical and computer engineering from the University of Michigan, Ann Arbor, in 1972.

Currently, he is a Professor of Electrical and Computer Engineering at the University of Wisconsin, Madison, and a Senior Consultant at Christensen Associates, Madison. He is also the Vice-Chairman of the IEEE Energy Policy Committee. He recently served as a member on a panel of experts for the study of a National Interest Transmission Grid for the U.S., reporting results to the Secretary of Energy in December 2001. He has developed methods for efficient trading in power networks, including a method for hedging against price uncertainty (with R. Rajaraman). He has done extensive work for CIGRE on the topic of ancillary services. He is a well-known software expert, with emphasis on large scale computation, sparse matrices, and least squares methods and co-author of several software packages. He has published many papers, articles, book chapters, reports, and conference presentations. He is recognized for his work on the integration of economics and electric power networks. He also participated in a Presidential Task Force on Critical Infrastructures during 1997 and 1998.

Dr. Alvarado is the Vice-Chair of the IEEE-USA Energy Policy Committee. He is a Fellow of IEEE. 